\title{
Quasi-periodic changes in the 3D solar anisotropy of Galactic cosmic rays for $1965-2014$
}

\author{
R. Modzelewska ${ }^{1}$ and M. V. Alania ${ }^{1,2}$ \\ 1 Institute of Math. and Physics, Siedlce University, 3 Maja 54 Street, 08-110 Siedlce, Poland \\ e-mail: renatam@uph.edu.pl \\ 2 Institute of Geophysics, Tbilisi State University, Tbilisi 380093, Georgia \\ e-mail: alania@uph.edu.pl
}

Received 2 August 2017 / Accepted 14 November 2017

\begin{abstract}
Aims. We study features of the 3D solar anisotropy of Galactic cosmic rays (GCR) for 1965-2014 (almost five solar cycles, cycles 20-24). We analyze the 27-day variations of the 2D GCR anisotropy in the ecliptic plane and the north-south anisotropy normal to the ecliptic plane. We study the dependence of the 27-day variation of the 3D GCR anisotropy on the solar cycle and solar magnetic cycle. We demonstrate that the 27 -day variations of the GCR intensity and anisotropy can be used as an important tool to study solar wind, solar activity, and heliosphere.

Methods. We used the components $A_{r}, A_{\varphi}$ and $A_{t}$ of the 3D GCR anisotropy that were found based on hourly data of neutron monitors (NMs) and muon telescopes (MTs) using the harmonic analyses and spectrographic methods. We corrected the 2D diurnal ( 24-h) variation of the GCR intensity for the influence of the Earth magnetic field. We derived the north-south component of the GCR anisotropy based on the $G G$ index, which is calculated as the difference in GCR intensities of the Nagoya multidirectional MTs. Results. We show that the behavior of the 27-day variation of the 3D anisotropy verifies a stable long-lived active heliolongitude on the Sun. This illustrates the usefulness of the 27-day variation of the GCR anisotropy as a unique proxy to study solar wind, solar activity, and heliosphere. We distinguish a tendency of the 22-yr changes in amplitude of the 27-day variation of the 2D anisotropy that is connected with the solar magnetic cycle. We demonstrate that the amplitudes of the 27-day variation of the north-south component of the anisotropy vary with the 11-yr solar cycle, but a dependence of the solar magnetic polarity can hardly be recognized. We show that the 27-day recurrences of the $G G$ index and the $A_{t}$ component are highly positively correlated, and both are highly correlated with the $B_{y}$ component of the heliospheric magnetic field.
\end{abstract}

Key words. Sun: activity - Sun: heliosphere - solar wind - Sun: rotation

\section{Introduction}

The flux of the Galactic cosmic rays (GCRs) measured at Earth consists of an isotropic and an anisotropic part. The isotropic part contains the various quasi-periodic changes with different timescales (from hours to several years), see, for instance, Kudela \& Sabbah (2016), Chowdhury et al. (2016), Bazilevskaya et al. (2014, and references therein), and 3D spatial density gradients (Kozai et al. 2014). The anisotropic part is generally reflected in the solar diurnal variation ( 24-h wave), assuming that while Earth completes one rotation, the location of a source of the anisotropic stream remains unchanged. The mechanism of the solar diurnal anisotropy was explained by Ahluwalia \& Dessler (1962) and also by Krymsky (1964) and Parker (1964), independently of each other, based on the anisotropic diffusion-convection theory of GCR propagation in the heliosphere. Chen \& Bieber (1993) have shed light on this problem assuming that the 3D GCR anisotropy is a combination of the 2D solar ecliptic and the north-south anisotropies. The 2D solar ecliptic anisotropy causes the daily variation in the count rate of ground-based detectors (e.g., neutron monitors (NMs) or muon telescopes (MTs)) that rotate with Earth; the north-south anisotropy reveals a flow of GCRs normal to the ecliptic plane. Swinson (1969) proposed that the north-south anisotropy might have occurred as a result of the drift that is caused by positive heliocentric radial density gradient $\boldsymbol{G}_{r}$ of cosmic rays and the $\boldsymbol{B}_{y}$ component of the heliospheric magnetic field (HMF; expressed as the vector product, $\boldsymbol{B}_{y} \times \boldsymbol{G}_{r}$ ).

Of recent publications devoted to the GCR anisotropy, we mention, for example, Kudela \& Sabbah (2016), Ahluwalia et al. 2015, Munakata et al. (2014), Sabbah (2013), and Oh et al. 2010. However, quasi-periodic changes of the GCR anisotropy connected with the solar rotation (called the 27-day variation here) were studied rarely until Alania et al. (2005, 2008) examined the ecliptic plane anisotropy (2D case) and the north-south component alone for polar-located NMs was considered by Owens et al. (1980). Swinson and coauthors (Swinson \& Yasue 1991; 1992; Swinson et al. 1993; Swinson \& Fuji 1995) reported the significant correlation between the 27-day variation of the north-south anisotropy for MT data and the tilt angle of the heliospheric neutral sheet. Alania et al. $(2005,2008)$ and Gil et al. (2012) analyzed the periods near the minima epochs of solar activity. They demonstrated that the amplitude of the 27 -day variation of the $2 \mathrm{D}$ anisotropy is smaller in the negativepolarity period than in the positive-polarity period of the HMF.

The main aim of this paper is twofold: (1) to investigate the 27-day variation of the 3D GCR solar anisotropy and its long-term changes during about five solar cycles, cycles 20-24; 


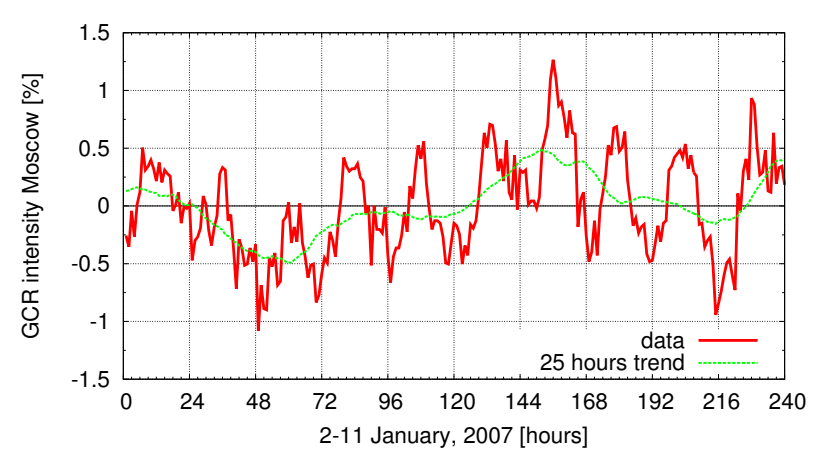

Fig. 1. Hourly data of Moscow NM in January 2-11, 2007 (red line) with the $25 \mathrm{~h}$ trend (green line).

and (2) to study the time lines of the 27-day variations of the north-south component of the GCR anisotropy and the 2D GCR anisotropy in the ecliptic plane.

\section{Data and methods}

We used data of the neutron monitors located at Kiel, Moscow, Oulu, Deep River, and Climax, which have magnetic cutoff rigidities of $R_{\mathrm{c}}<5 \mathrm{GV}$ to clearly reveal the 3D GCR anisotropy. The pressure-corrected hourly data of the GCR intensity were normalized as $I[\%]=\frac{x_{i}-\bar{x}}{\bar{x}}$, where $\bar{x}$ is average count rate of the observed GCR intensity. We excluded trends larger than the diurnal variation $(24 \mathrm{~h}$ ) using the 25 -h moving-average method. As an example, we present the hourly data of the Moscow NM for January 2-11, 2007, in Fig. 1, and the same "detrended" data in Fig. 2. Figures 1 and 2 demonstrate that the GCR intensity (Fig. 1) alternates under the influence of a trend with a period longer than $25 \mathrm{~h}$, while the "detrended" data (Fig. 2) show an intensity fluctuation of about zero, which is essential for a harmonic analysis.

We calculated the daily radial $a_{r}$ and tangential $a_{\varphi}$ components of the diurnal variation of the GCR intensity by normalized and detrended hourly data of the GCR intensity using the harmonic analyses method (e.g., Gubbins 2004):

$$
\begin{aligned}
I(t) & =\frac{a_{0}}{2}+\sum_{k=1}^{\infty}\left(a_{r}^{k} \cos \frac{2 \pi k t}{T}+a_{\varphi}^{k} \sin \frac{2 \pi k t}{T}\right) \\
& =\sum_{i=1}^{\infty} a_{k} \sin \left(\frac{2 \pi k t}{T}+\varphi_{k}\right) .
\end{aligned}
$$

Here,

$a_{r}^{k}=\frac{1}{p} \sum_{i=1}^{2 p} x_{i} \cos \frac{\pi k i}{p}, \quad a_{\varphi}^{k}=\frac{1}{p} \sum_{i=1}^{2 p} x_{i} \sin \frac{\pi k i}{p}, \quad a_{0}=\frac{1}{p} \sum_{i=1}^{2 p} x_{i}$, $\varphi_{k}=\operatorname{arctg} \frac{a_{\varphi}^{k}}{a_{r}^{k}}, \quad a_{k}=\sqrt{a_{r}^{2}+a_{\varphi}^{2}}$

$2 p=24 \mathrm{~h}$, and $x_{i}$ designates the hourly data of the GCR intensity for each NM. We corrected the daily radial $a_{r}$ and tangential $a_{\varphi}$ components of the diurnal variation of the GCR intensity for the influence of the terrestrial magnetic field (Rao et al. 1963; Dorman et al. 1972; Dorman 2009), taking into account the asymptotic acceptance cone that is characteristic for each NM. This was done by rotating the corresponding angle $\lambda$ (asymptotic longitude) for each NM. We calculated the radial $a_{r}^{E}$ and the tangential $a_{\varphi}^{E}$ components of the diurnal variation of the



Fig. 2. Same data as in Fig. 1 "detrended" with the 25 h.

GCR intensity corrected for the influence of the terrestrial magnetic field using the expressions

$a_{r}^{E}=a_{r} \cos \lambda-a_{\varphi} \sin \lambda \quad a_{\varphi}^{E}=a_{r} \sin \lambda+a_{\varphi} \cos \lambda$.

Furthermore, we excluded from consideration the amplitudes $>0.7 \%$ as an anomalous event related to the disturbances in interplanetary space, generally corresponding to the periods of Forbush decreases. Fewer than $2-3 \%$ of the total number of days were excluded. The amplitude of the diurnal variation of the GCR intensity $a_{j}^{E}$ at any point of the observation (by a NM) with the geomagnetic cutoff rigidity $R_{\mathrm{c}}$ and the average atmospheric depth $h_{j}$ can be defined as

$a_{j}^{E}=\int_{R_{\mathrm{c}}}^{R_{\max }} \frac{\delta D(R) W\left(R, h_{j}\right)}{D(R)} \mathrm{d} R$,

where $\frac{\delta D(R)}{D(R)}$ is the rigidity spectrum of the diurnal variation of the GCR intensity, and $W\left(R, h_{j}\right)=\frac{D(R)}{N} m\left(R, h_{j}\right)$ is the coupling function (Dorman 1963; Yasue et al. 1982); $R_{\max }$ is the upper limit of the rigidity beyond which the amplitudes of the diurnal variation of the GCR intensity vanish. For the power type of the rigidity spectrum $\frac{\delta D(R)}{D(R)}=A R^{-\gamma}$, we can write

$A_{j}=\frac{a_{j}^{E}}{\int_{R_{\mathrm{c}}}^{R_{\max }} R^{-\gamma} W\left(R, h_{j}\right) \mathrm{d} R}$,

where $a_{j}^{E}$ is the observed amplitude of the diurnal variation of the GCR intensity for $j$ th $\mathrm{NM}$, and $A_{j}$ is the corresponding amplitude of the anisotropy of GCRs in the heliosphere. The values of $A_{j}$ must be the same in the scope of the calculation accuracy for arbitrary NM data when the parameter pairs $\gamma$ and $R_{\max }$ are properly determined. The similarity of the $A_{j}$ values we found for the different NMs is a decisive factor that confirms that the data of the given NM are reliable. We converted the radial $a_{r}$ and azimuthal $a_{\varphi}$ components of the diurnal GCR variation into the radial $A_{r}$ and azimuthal $A_{\varphi}$ components of the GCR anisotropy in the heliosphere (free space; Dorman 1963; Yasue et al. 1982),

$$
\begin{aligned}
A_{r_{j}}= & \frac{a_{r_{j}}^{E}}{\int_{R_{\mathrm{c}}}^{R_{\max }} R^{-\gamma} W\left(R, h_{j}\right) \mathrm{d} R} \\
A_{\varphi_{j}}= & \frac{a_{\varphi_{j}}^{E}}{\int_{R_{\mathrm{c}}}^{R_{\max }} R^{-\gamma} W\left(R, h_{j}\right) \mathrm{d} R},
\end{aligned}
$$

where the expression $\int_{R_{\mathrm{c}}}^{R_{\max }} R^{-\gamma} W\left(R, h_{j}\right) \mathrm{d} R$ is called the coupling coefficient (CC). This is the ratio of the observed amplitude 
Table 1. Details of the neutron monitors and values of corresponding coupling coefficients $(C C)$ vs. solar activity.

\begin{tabular}{ccccclll}
\hline \hline NM station & latitude & longitude & $R_{\mathrm{c}}[\mathrm{GV}]$ & $\lambda\left[^{0}\right]$ & $\mathrm{CC}$ minimum & CC intermediate & CC maximum \\
\hline Climax & $39.40 \mathrm{~N}$ & $253.8 \mathrm{E}$ & 3.03 & -67 & 0.705 & 0.7105 & 0.716 \\
Deep River & $46.10 \mathrm{~N}$ & $-77.5 \mathrm{~W}$ & 1.14 & -44 & 0.715 & 0.7215 & 0.728 \\
Kiel & $54.30 \mathrm{~N}$ & $10.1 \mathrm{~W}$ & 2.36 & 60 & 0.659 & 0.6355 & 0.612 \\
Moscow & $55.50 \mathrm{~N}$ & $37.3 \mathrm{E}$ & 2.43 & 84 & 0.661 & 0.6380 & 0.615 \\
Oulu & $65.05 \mathrm{~N}$ & $25.47 \mathrm{E}$ & 0.8 & 62 & 0.619 & 0.5895 & 0.560 \\
\hline
\end{tabular}

of the diurnal variation to the corresponding amplitude of the anisotropy of cosmic rays in the heliosphere. We provide a conversion for the same set of parameters as in Bieber \& Chen (1991), namely $R_{\max }=100 \mathrm{GV}$ and spectral index $\gamma=0$. This selection of the parameters $R_{\max }$ and $\gamma$ is reasonable because the criterion of equality of the $A_{j}$ values (the amplitudes of the GCR anisotropy in the heliosphere) found for the different NMs is satisfied. We found that for $\gamma=0.5$, there are no great changes in the results, meaning that the rigidity dependence of the anisotropy is reasonably weak for the energy range of GCR particles to which the NMs respond; a hard GCR anisotropy spectrum was found in Hall et al. (1996).

The details of the NMs we used and the corresponding $C C$ values versus solar activity are presented in Table 1.

We also used results of the 3D GCR anisotropy calculated by the group of Institute of Terrestrial Magnetism, Ionosphere and Radio Wave Propagation of the Russian Academy of Sciences (IZMIRAN; Abunina et al. 2015; Belov et al. 2005) by applying the global spectrographic method (GSM; Krymsky et al. 1966; 1967) which contains all operating NMs. Furthermore, to study the features of the 3D GCR anisotropy in the relatively wide range of the GCR spectrum, we used data from the Nagoya MTs (Munakata et al. 2014) with a median rigidity of $\sim 60 \mathrm{GV}$.

We used the power spectrum density (PSD) method to reveal the quasi-periodicity in the analyzed time series; this method decomposes the time series into frequency $(\omega)$ and period $(T)$ components (e.g., Otnes \& Enochson 1972; Press et al. 2002),

$\psi(\omega)=\int_{-\infty}^{\infty} R(t) \mathrm{e}^{-\mathrm{i} \omega t} \mathrm{~d} t$

where $R(t)$ is the autocorrelation function. In a discrete case, we have

$\psi(\omega)=\Delta t \sum_{r=-N}^{N} W \cdot R(r) \mathrm{e}^{-\mathrm{i} \omega r \Delta t}$

here $R(r)=\frac{1}{N-r} \sum_{i=1}^{N-r} x_{i} x_{i+r}$ is the autocorrelation function of the time series $x_{i}$ and $W$ is the window function (we used Parzen's window function). The PSD of each frequency $\omega$ is calculated as $\left|\psi^{2}(\omega)\right| H z^{-1}$.

\section{27-day variation of the 2D GCR anisotropy in the ecliptic plane}

Unfortunately, a complete precise method for a simultaneous calculation of the radial $A_{r}$, azimuthal $A_{\varphi}$ and latitudinal (northsouth) $A_{t}$ components of the 3D GCR anisotropy based on the worldwide network of NMs and MTs is currently not available. The $A_{r}$ and $A_{\varphi}$ components can be calculated using the GSM and harmonic analyses methods, which are based on data of the NMs with cutoff rigidities $<5 \mathrm{GV}$; in addition, a latitudinal $A_{t}$ (north-south) component can be calculated within the scope of the arbitrary constant, but only with the GSM method. Additionally, the $A_{t}$ component can be estimated as the difference of two NMs that are located in the regions of the north and south poles (Chen \& Bieber 1993), and $A_{t}$ can also be estimated by directed MTs. However, these data are not homogenous, and to study features of 3D anisotropy (e.g., the 27-day variation of the 3D anisotropy), we have to use results for $A_{r}, A_{\varphi}$ and $A_{t}$ that are obtained in different ways.

The 27-day variations of the GCR intensity (e.g., Richardson et al. 1999; Dunzlaff et al. 2008; Guo \& Florinsky 2014; 2016; Kopp et al. 2017; Gil \& Mursula 2017) and the anisotropy (e.g., Modzelewska \& Alania 2012; Mavromichalaki et al. 2016) have a sporadic character. Their amplitudes significantly increase and decrease on average during four to six solar rotation periods. However, they are not completely random phenomena. Generally, some levels of the 27-day variation amplitudes of the GCR intensity and anisotropy always exist that are above the background fluctuations. This statement is demonstrated in Figs. 3-5, where we present the changes in GCR intensity (Fig. 3), and we also show the $A_{r}$ (Fig. 4) and $A_{\varphi}$ (Fig. 5) components of the GCR anisotropy for the period of 2007-2009 for the Oulu NM. We present in Figs. 3-5 the corresponding data "detrended" by moving smoothing intervals of 29 days and a filtered periodic oscillation with the bandpass period within 24-32 days. Figures 3-5 show periods with a well-pronounced 27-day recurrence with high amplitudes of the 27-day GCR intensity variations and the anisotropy components, as well as very small amplitudes in other periods. This behavior of the 27-day variations of the GCR intensity and solar anisotropy is related with some disparate mechanisms. The 27-day variation of the GCR intensity is mainly connected with heliolongitudinal asymmetry of the solar wind and the solar activity and their dependences on heliolatitudes. This means that the 27-day variation in GCR intensity contributes a fairly large part of interplanetary space. At the same time, the convection-diffusion mechanism of the solar anisotropy (Krymsky 1964; Parker 1964) is determined by local processes near the Earth orbit and requires a smaller part of the heliosphere. Above all, especially in the solar activity minima epochs, the 27-day variation in anisotropy is rarely observed, which is apparently connected with the drift of cosmic rays in the sector structure of the HMF, while a weak 27-day variation in GCR intensity is observed. The circumstances can be reversed when a clear 27-day GCR intensity variation is observed that does not follow the 27-day anisotropy variation at all. Unfortunately, this phenomenon is currently not fully explained, which indicates that the problem of the 27-day anisotropy and intensity variations is not fully understood so far. No universal mechanism of the 27-day GCR anisotropy and intensity variations is currently available that would account for dynamical changes in features in solar atmosphere and heliosphere sources. Therefore we need to find new properties of the 27-day GCR anisotropy and intensity. This problem is one of the important aims of this paper. 


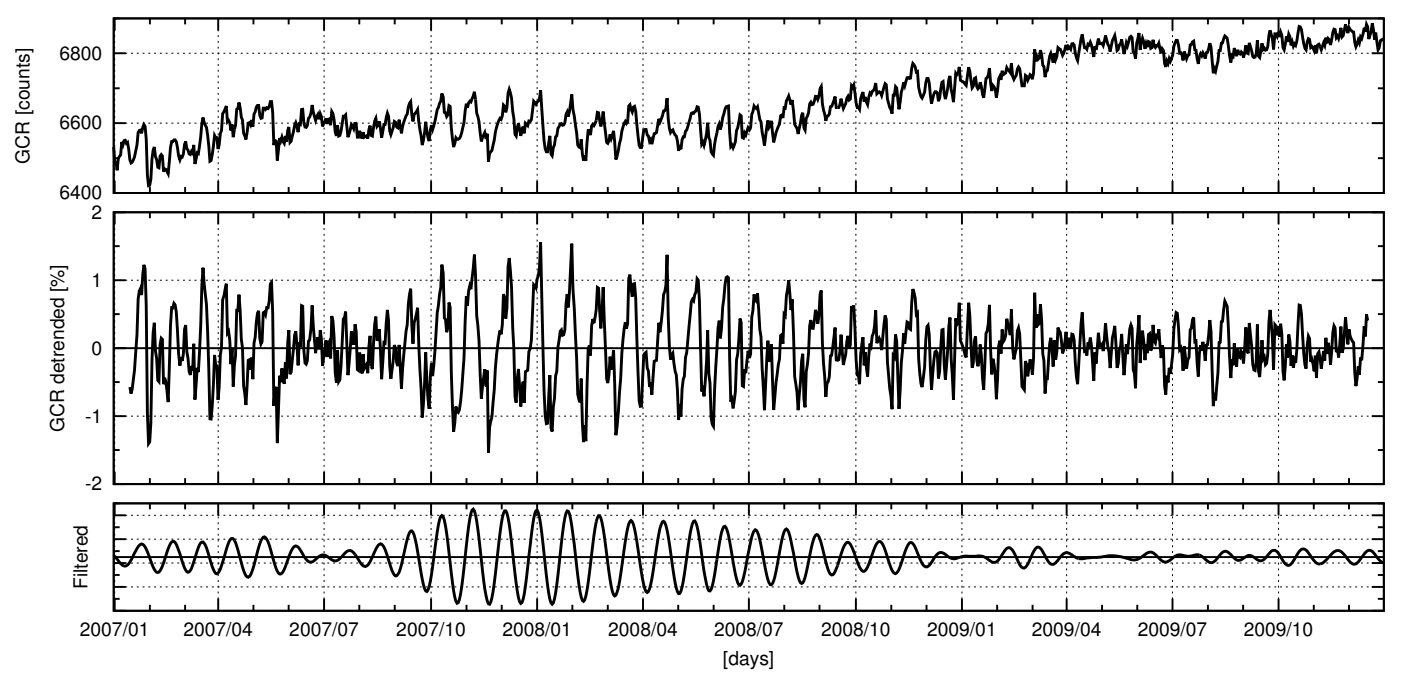

Fig. 3. Temporal changes of the daily GCR intensity for Oulu NM for the period of 2007-2009 (top) and corresponding to this period the same data detrended over 29 days (middle) and filtered periodic oscillation with the band-pass period within 24-32 days (bottom).

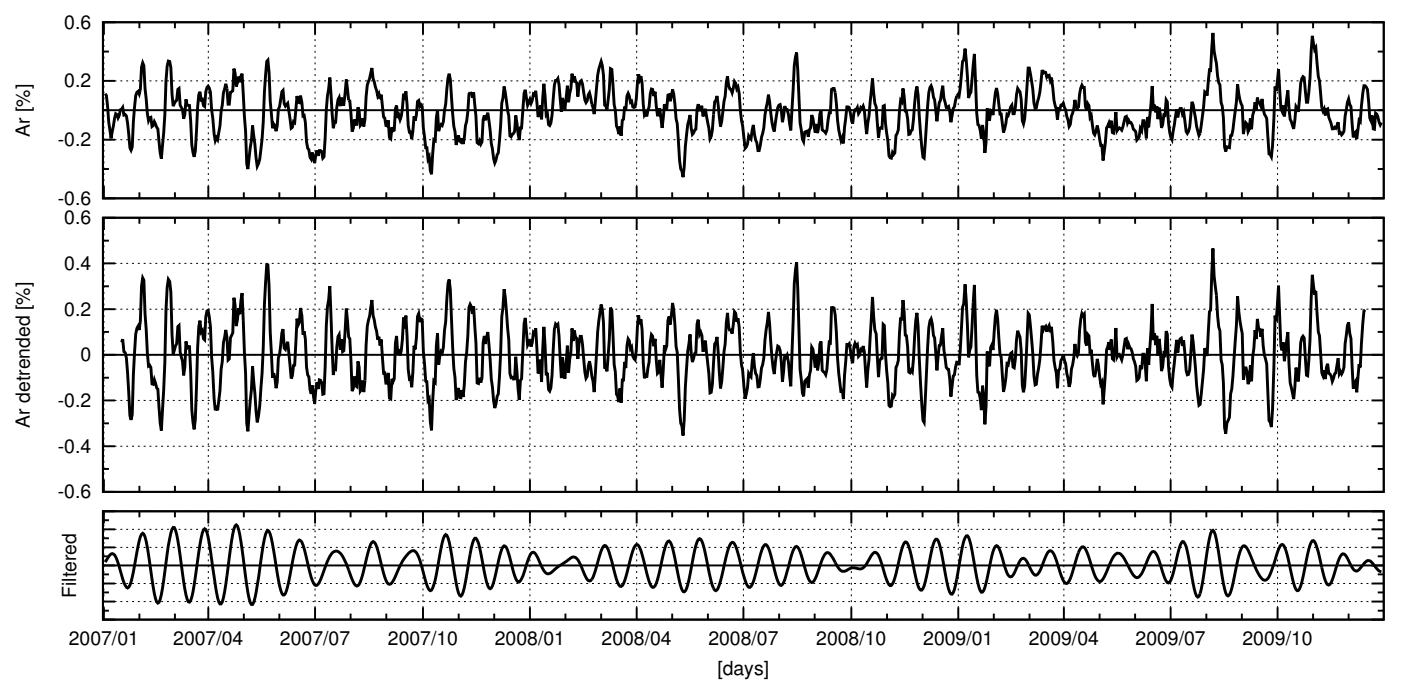

Fig. 4. Same as in Fig. 3 but for $A_{r}$ component of the GCR anisotropy for Oulu NM.

Taking the sporadic nature of the 27-day GCR anisotropy variation into account (it lasts on average for about four to six solar rotations), studying its behavior for the long period of 1971-2014 is naturally interesting. For this purpose, we applied the spectral analysis method based on the Oulu NM data. The calculation results are presented in Fig. 6. Figure 6 presents the spectral analysis of the daily radial $A_{r}$, the azimuthal $A_{\varphi}$ components, and of the phase $=\arctan \left(A_{\varphi} / A_{r}\right)$ of the 2D GCR anisotropy for the Oulu NM for the long period of 1971-2014. Figure 6 shows that the $A_{r}$ and $A_{\varphi}$ components and phase apparently demonstrate quasi-periodic changes related to the Sun's rotation. For the components $A_{r}$ and $A_{\varphi}$ and for the phase, the highest peaks correspond to a period of 25.6 days with a confidence level of $95 \%$. Figure 6 shows stable long-lived active heliolongitudes on the Sun that we consider as the source of the 27-day GCR anisotropy variation.

\section{27-day variation of the north-south anisotropy}

To study the 27-day variation of the north-south anisotropy, we used two types of data: (1) the daily $A_{t}$ component calculated with the GSM for NMs data ${ }^{1}$, and (2) the daily $G G$ index obtained for $\mathrm{MTs}^{2}$. The $G G$ index (Mori \& Nagashima 1979) is the difference between the intensities that are recorded in the geographically north $(N 2)$ south $(S 2)$ and east (E2)-viewing directional channels for 49 degrees inclination, corresponding to a median rigidity of $\sim 60 \mathrm{GV}$ and calculated as follows: $G G=$ $(N 2-S 2)+(N 2-E 2)$.

This method for studying the north-south asymmetry has the advantage that it uses the GCR intensity data from a single location instead of comparing GCR intensities from north and south polar NMs. The $G G$ index as introduced according to Mori \& Nagashima (1979) is expected to be free of noise in isotropic intensity caused by Forbush decreases, periodic variations, atmospheric temperature effects, and geomagnetic cutoffs. Although the counting rate of GCR intensities in different directions ( $N 2, S 2$, and $E 2)$ cannot contain exactly the same type of information, the $G G$ is accepted as a good alternative index by the worldwide cosmic ray community (e.g., Munakata et al. 2014). Recently, we analyzed (Modzelewska \& Alania 2015)

\footnotetext{
http://cr0.izmiran.ru/CosmicRayAnisotropy/ http://www.stelab.nagoya-u.ac.jp/
} 


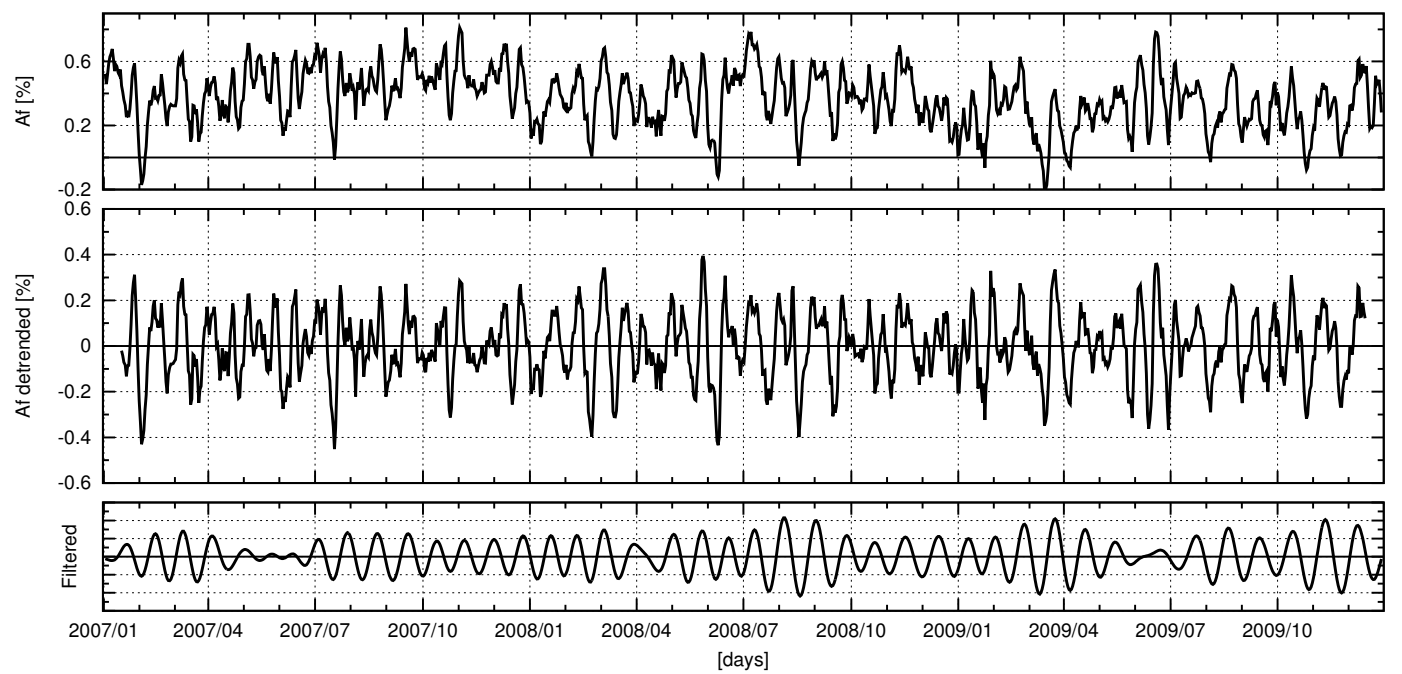

Fig. 5. Same as in Fig. 3 but for $A_{\varphi}$ component of the GCR anisotropy for Oulu NM.
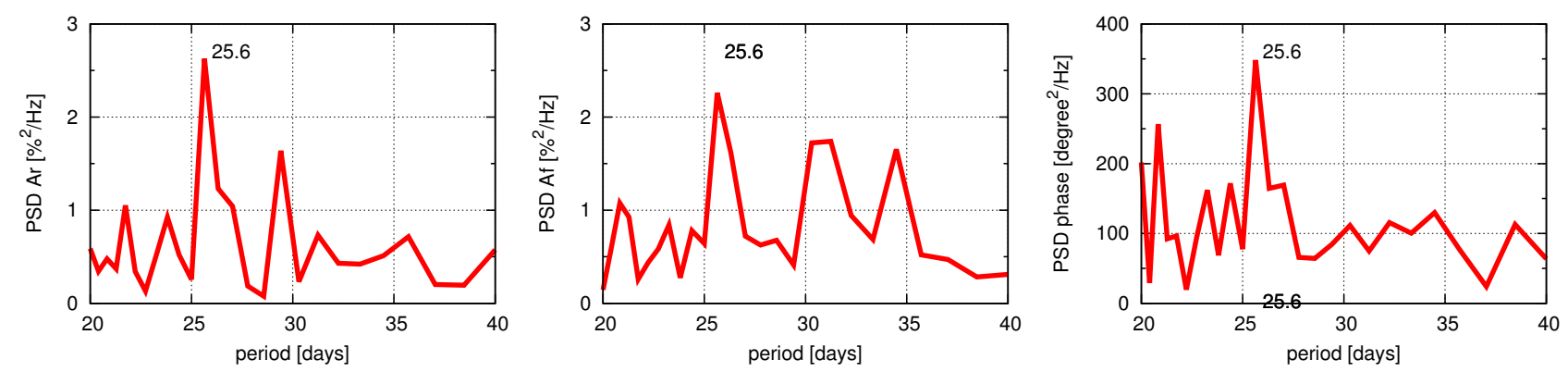

Fig. 6. Spectral analysis of the daily radial $A_{r}$ (left panel), azimuthal $A_{\varphi}$ (middle panel) and phase (right panel) of the 2D GCR anisotropy for Oulu NM for 1971-2014, for all the highest peak is for the period of 25.6 days with $95 \%$ confidence level.

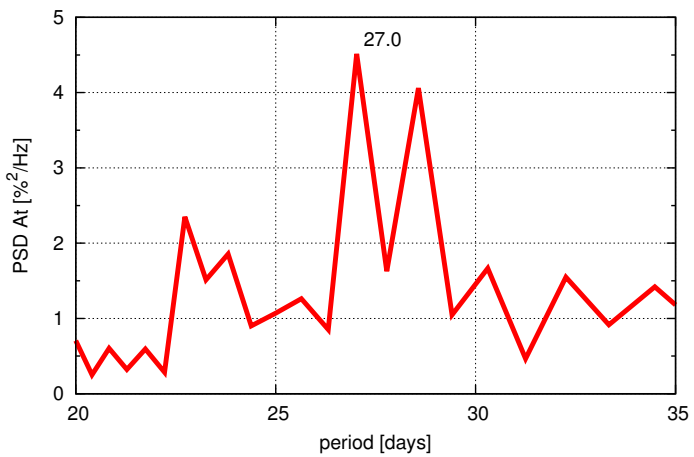

Fig. 7. Spectral analysis of the $A_{t}$ component of the 3D GCR anisotropy for 1970-2006, the highest peak is for the period of 27 days with $95 \%$ confidence level.

the behavior of the quasi-periodic changes of the $G G$ index for 2007-2012 with the wavelet time-frequency method. In this paper we extend this study of the $G G$ index and the $A_{t}$ anisotropy component for 1971-2014 with the PSD method.

We present the results of analysis in Figs. 7 and 8. Figure 7 shows the results of the spectral analysis of the $A_{t}$ component of the 3D GCR anisotropy for 1971-2006 (data of $A_{t}$ are available at the IZMIRAN website until 2006) and Fig. 8 of the $G G$ index for 1971-2014. Figures 7 and 8 show that the $A_{t}$ component and the $G G$ index both apparently demonstrate quasi-periodic changes that are related to the Sun's rotation. For the $A_{t}$ component, the period is 27 days and for the $G G$ index, it is 26.3 days with a confidence level of 95\%. In Figs. 6 (left panel), 7, and 8,

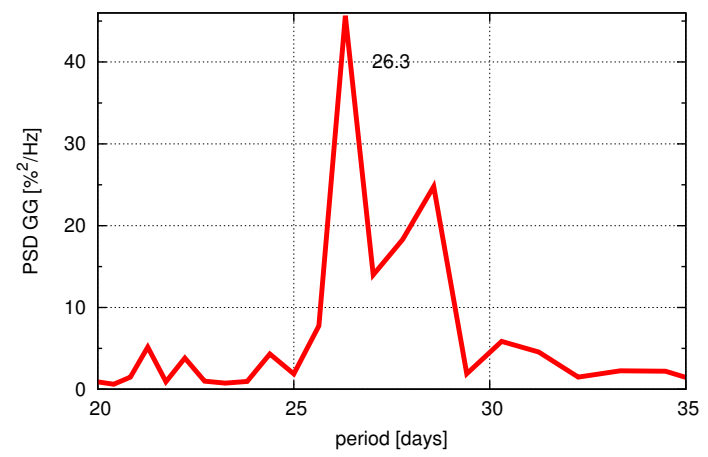

Fig. 8. Spectral analysis of the daily $G G$ index for 1971-2014, the highest peak is for the period of 26.3 days with $95 \%$ confidence level.

peaks at $\sim 28-29$ days are visible, but they are statistically insignificant. We do not exclude the presence of an additional source with an average period of 28-29 days, however, that is due to the differential rotation of the Sun, which manifests itself as the asymmetry of heliolongitudes versus heliolatitudes.

\section{Long-period changes of the 27-day variation amplitudes}

\section{1. $2 D$ ecliptic plane anisotropy}

To study the long-period changes of the 27-day variation amplitudes of the 2D ecliptic plane GCR anisotropy, we calculated the 2D 27-day variation amplitudes of the anisotropy (A27A) 


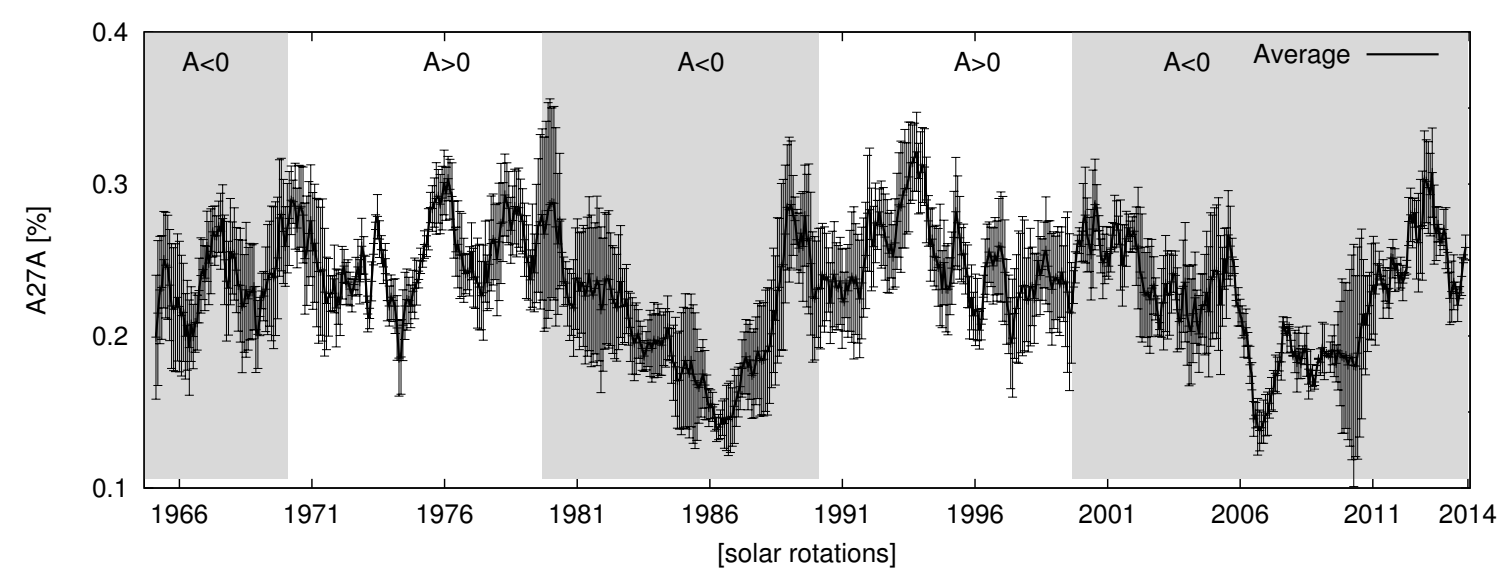

Fig. 9. Temporal changes of the average $A 27 A$ smoothed over 13 solar rotations for all considered NMs (Moscow, Kiel, Oulu, Deep River, Climax) for 1965-2014 during $A>0$ and $A<0$ polarity epochs; error bars are calculated as standard deviations for considered NMs.

for the Climax, Deep River, Kiel, Moscow, and Oulu NM data for 1965-2014. We used the formula (Alania et al. 2005) that combines the 27-day recurrence of the radial and azimuthal components:

$$
A 27 A=\sqrt{\left(A_{r r}(27)+A_{\varphi r}(27)\right)^{2}+\left(A_{r \varphi}(27)+A_{\varphi \varphi}(27)\right)^{2}}
$$

Here $A_{r r}(27)$ and $A_{r \varphi}(27)$ are the coefficients of the 27-day variation of the $A_{r}$ component, and $A_{\varphi r}(27)$ and $A_{\varphi \varphi}(27)$ are the coefficients of the 27-day variation of the $A_{\varphi}$ component.

We present the calculation results in Fig. 9. Figure 9 shows the temporal changes in the average amplitude of the 27-day variation of the 2D GCR anisotropy in the ecliptic plane smoothed over 13 solar rotations for the Climax, Deep River, Kiel, Moscow, and Oulu NMs in the time interval 1965-2014; the error bars are calculated as standard deviations for the NMs. The smoothing over 13 solar rotations reduces the error bars, but at the same time, we lost information about the quasi-periodic changes for a time interval of slightly less than one year.

Figure 9 shows that the average amplitude of the 27-day variation of the GCR 2D anisotropy is larger in the minimum periods of the positive-polarity $(A>0)$ epochs than in the negative $(A<0)$ epochs, which agrees well with Alania et al. (2005, 2008). In addition, the time line of the amplitudes of the 27-day variation of the 2D GCR anisotropy shows a tendency of the 22-yr periodicity and a weak but systematic decrease near the periods of solar minimum for an $A>0$ polarity ( $\sim 1975$ and $\sim 1996$ ) as well, indicating a weak 11-yr solar cycle modulation. The ecliptic 2D anisotropy components strongly reflect the drift pattern of the cosmic ray flow, and the polarity dependence is consequently apparent in the 27-day variation of the 2D GCR anisotropy.

To reveal a polarity dependence of the 27-day variation of the 2D GCR anisotropy, we calculated the differences in amplitudes between the $A>0$ and $A<0$ polarities, which is equal to $\sim 40 \%$ of the mean value. In particular, the average amplitude of the 27-day variation of the 2D GCR anisotropy A27A for the whole period $1965-2014$ is $(0.23 \pm 0.04) \%$, and the values of $A 27 A$ for the consecutive minima are

for $A>01975-1977 A 27 A=(0.26 \pm 0.01) \%$,

for $A<01985-1987 A 27 A=(0.16 \pm 0.01) \%$,

for $A>01995-1997 A 27 A=(0.24 \pm 0.02) \%$, and

for $A<0$ 2007-2009 $A 27 A=(0.18 \pm 0.02) \%$.

\subsection{Long-period changes of the 27-day variation amplitudes of the north-south anisotropy}

Recently, we showed (Modzelewska \& Alania 2015) that the daily $G G$ index is inversely related with the daily $B_{y}$ component of the HMF for 2007-2012. This effect, according to Swinson's (1969) formulation of the north-south anisotropy, is caused by drift, which we consider an acceptable explanation. To study the nature of the north-south asymmetry, we calculated the 27-day variation amplitudes of the $G G$ index $(A 27 G G), A_{t}\left(A 27 A_{t}\right)$ and $B_{y}$ components $\left(A 27 B_{y}\right)$ with the harmonic analysis method for 1971-2014. We present the results in Fig. 10. Figure 10 shows the temporal changes of $A 27 G G$ and $A 27 A_{t}$ (top), and $A 27 B_{y}$ (bottom) for 1970-2014. The behavior of the amplitudes of the 27-day variations of the $G G$ index and $A_{t}$ component is similar and highly positively correlated with the 27 -day variation of the $B_{y}$ component; all are alternating according to the 11-yr solar cycle. In contrast to the GCR anisotropy in the ecliptic plane, the north-south component has no clear magnetic polarity dependence (e.g., Munakata et al. 2014), so its 27-day recurrence is not expected to be dependent on the magnetic polarity either, which we observe in experimental data.

\section{Conclusions}

We studied the long-term changes of the 27-day variation amplitudes of the 3D GCR anisotropy for 1965-2014. We showed that the behavior of the 27-variation of the 3D anisotropy verifies the existence of a stable long-lived active heliolongitude on the Sun. This shows that the GCR anisotropy variation is useful as a unique proxy to study the solar wind, solar activity, and the heliosphere. We recovered a tendency of the 22-yr changes of the 27-day variation amplitudes of the 2D anisotropy to be connected with the solar magnetic cycle and a weak 11-yr solar cycle modulation. We demonstrated that the 27-day variation amplitudes of the north-south component of the anisotropy vary in accordance with 11-yr solar cycle, while it is hardly possible to show any dependence on the global solar magnetic field polarity. The 27-day recurrences of the $G G$ index and $A_{t}$ component are highly positively correlated, and both are highly correlated with the $B_{y}$ component of the HMF. The observed properties of the radial $A_{r}$, azimuthal $A_{\varphi}$, and normal $A_{t}$ components and of the $G G$ index demonstrate that quasi-periodic behaviors of the ecliptic 2D and north-south components of the 3D GCR anisotropy are governed by different mechanisms in the ecliptic plane and in 

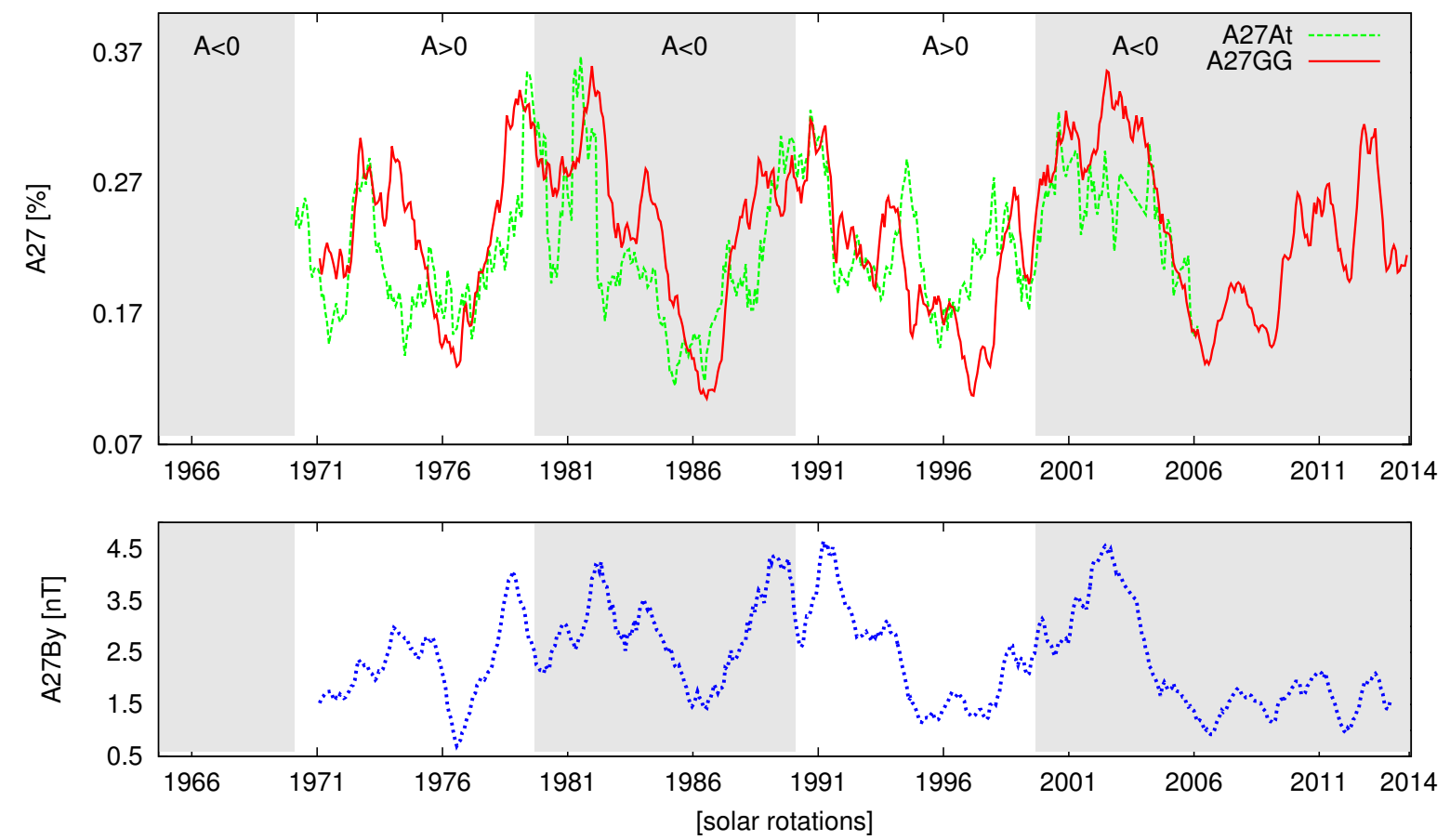

Fig. 10. Temporal changes of the amplitudes of the 27-day variation of the $G G$ index $(A 27 G G)$ and $A_{t}$ component $\left(A 27 A_{t}\right)$ of the $3 \mathrm{D}$ anisotropy (top) and $B y$ component of the HMF $\left(A 27 B_{y}\right.$ ) (bottom) smoothed over 13 Sun's rotations during $A>0$ and $A<0$ polarity epochs. Values $A 27 G G$ and $A 27 B_{y}$ are presented for 1971-2014, but $A 27 A_{t}$ - for 1970-2006, data of $A_{t}$ is available at the IZMIRAN website up to 2006.

the north-south direction. Owing to the complexity of the problem, we are unable to fully explain the physical mechanism of the 27-day anisotropies in 3D space. We are unable to completely clarify the processes related to the 27-day variations of the 3D anisotropy with indipendent theories or experimental data of NMs and MTs. For this purpose, theoretical modeling based on Parker's transport equation and experimental data have to be considered together.

Acknowledgements. We thank the providers of the data we used in this study, especially the OMNI database, the IZMIRAN group, and the PIs of the Oulu, Moscow, Kiel, Deep River, and Climax NMs and of the Nagoya MTs. R. Aslamazishvili, a member of M. Alania's scientific group in Tbilisi, participated in calculating the 2D ecliptic anisotropy components of GCRs within the asymptotic acceptance cone in Earth's magnetic field. The remarks and suggestions of the referee helped us to improve the paper.

\section{References}

Abunina, M., Abunin, A., Belov, A., et al. 2015, J. Phys.: Conf. Ser. 632, 012044 Ahluwalia, H. S., \& Dessler, A. J. 1962, Planet. Space Sci., 9, 195

Ahluwalia, H. S., Ygbuhay, R. C., Modzelewska, R., et al. 2015, J. Geophys. Res., 120, 8229

Alania, M. V., Gil, A., Iskra, K., et al. 2005, in Proc. 29th ICRC, SH3.4, 215

Alania, M. V., Gil, A., \& Modzelewska, R. 2008, Adv. Space Res., 41, 280

Bazilevskaya, G., Broomhall, A.M., Elsworth, Y., \& Nakariakov, V. M. 2014, Space Sci. Rev., 186, 359

Belov, A. V., Baisultanova, L., Eroshenko, E. A., et al. 2005, J. Geophys. Res., 110, A09S20

Bieber, J. W., \& Chen, J. 1991, ApJ, 372, 301

Chen, J., \& Bieber, J. W. 1993, ApJ, 405, 375

Chowdhury, P., Kudela, K., \& Moon Y. J. 2016, Solar Phys., 291, 581

Dorman, L. I. 1963, Variations of cosmic rays and space exploration (AN SSSR Moscow)

Dorman, L. I. 2009, Cosmic Rays in Magnetospheres of the Earth and other Planets, Astrophys. Space Sci. Lib., 358 (The Netherlands: Springer)

Dorman, L. I., Gushchina, R. T., Smart, D. F., \& Shea, M. A. 1972, Effective Cut-Off Rigidities of Cosmic Rays (Moscow: Nauka)

Dunzlaff, P., Heber, B., Kopp, A., et al. 2008, Ann. Geophys., 26, 3127
Gil, A., \& Mursula, K. 2017, A\&A, 599, A112

Gil, A., Modzelewska, R., \& Alania, M. V. 2012, Adv. Space Res., 50, 712 Gubbins, D. 2004, Time Series Analysis and Inverse Theory for Geophysicists (Cambridge: Cambridge University Press), 21

Guo, X., \& Florinski, V. 2014, J. Geophys. Res.: Space Phys., 119, 2411

Guo, X., \& Florinski, V. 2016, ApJ, 826, 65

Hall, D. L., Duldig, M. L., \& Humble, J. E. 1996, Space Sci. Rev., 78, 401

Kopp, A., Wiengarten, T., Fichtner, H., et al. 2017, ApJ, 837, 37

Munakata, K., Kato, Ch., Kuwabara, T., et al. 2014, Earth, Planets and Space, 66, 151

Krymsky, G. F. 1964, Geomagnetism \& Aeronomy, 4, 763

Krymski, G. F., Kuzmin, A. I., Chirkov, N. P., et al. 1966, Geomagnetism \& Aeronomy, 6, 991

Krymski, G. F., Kuzmin, A. I., Chirkov N. P., et al. 1967, Geomagnetism \& Aeronomy, 7, 11

Kudela, K., \& Sabbah, I. 2016, Sci. Ch. Technol. Sci., 59, 547

Mavromichalaki, H., Papageorgiou, Ch., \& Gerontidou, M. 2016, Astrophys. Space Sci., 361, 69

Modzelewska, R., \& Alania, M. V. 2012, Adv. Space Res., 50, 716

Modzelewska, R., \& Alania, M. V. 2015, J. Phys.: Conf. Ser., 632, 012072 , 1742

Mori, S., \& Nagashima, K. 1979, Planet. Space Sci., 27, 39

Munakata, K., Kozai, M., Kato, C., \& Kota, J. 2014, ApJ, 791, 22

Oh, S. Y., Yi, Y., \& Bieber, J. W. 2010, Sol. Phys., 262, 199

Otnes, R. K., Enochson, L. 1972, Digital Time Series Analysis (New York: John Wiley and Sons), 191

Owens, A. J., Duggal, S. P., Pomerantz, M. A., \& Tolba, M. F. 1980, ApJ, 236, 1012

Parker, E. N. 1964, Planet. Space Sci., 12, 735

Press, W. H., Teukolsky, S. A., Vetterling, W. T., \& Flannery, B. P. 2002, The Art of Scientific Computing (Cambridge University Press), 550

Rao, U. R., McCracken, K. G., \& Venkatesan, D. 1963, J. Geophys. Res., 68, 345

Richardson, I. G., Cane, H. V., \& Wibberenz, G. 1999, J. Geophys. Res., 104, 12549

Sabbah, I. 2013, J. Geophys. Res., 118, 4739

Swinson, D. B. 1969, J. Geophys. Res., 74, 5591

Swinson, D. B., \& Yasue, S. I. 1991, in Proc. 22nd ICRC, SH, 481

Swinson, D. B., \& Yasue, S. I. 1992, J. Geophys. Res., 92, A12, 19149

Swinson, D. B., Yasue, S., \& Fujii, Z. 1993, in Proc. 23rd ICRC, 671

Swinson, D. B., \& Fujii, Z. 1995, in Proc. 24th ICRC, SH, 576

Yasue, S., Sakakibara, S., \& Nagashima, K. 1982, Coupling coefficients of cosmic rays daily variations for neutron monitors (Nagoya Report), 7 